\title{
Avifauna em Quatro Fazendas Madeireiras de Irati-PR: Análise para Certificação Florestal
}

\author{
Vânia Rossetto Marcelinoํㅜㄹ Kelly Geronazzo Martins² \\ ${ }^{1}$ Departamento de Engenharia Florestal - DEF, Universidade Estadual do Centro-Oeste - UNICENTRO, Irati/PR, Brasil \\ ${ }^{2}$ Departamento de Engenharia Ambiental - DENAM, Universidade Estadual do Centro-Oeste - UNICENTRO, Irati/PR, Brasil
}

\begin{abstract}
RESUMO
O presente estudo comparou a comunidade e a diversidade de aves em fragmentos de Floresta Ombrófila Mista de diferentes tamanhos. Para tanto, oito fragmentos divididos em conjuntos, formando duas classes (somando de 20 a 70 ha e de 100 a 420 ha), foram selecionados em quatro fazendas no distrito de Guamirim, Irati-PR. O método de amostragem consistiu em trajetos irregulares percorridos pelas áreas naturais, realizados entre 2009 e 2010, sempre na primavera e no outono. Foram calculadas a Frequência Relativa e a Diversidade mediante o índice de Shannon. As análises MRPP e Cluster, e o teste Mann-Whitney (Wilcoxon) W foram utilizados para comparar os fragmentos. Os resultados comprovam que fragmentos maiores abrigam uma comunidade distinta e mais diversa do que manchas menores do que 100 ha. Foram registradas 88 espécies pelos trajetos irregulares. Somando estas às espécies anotadas a partir de observações eventuais em qualquer área dentro das fazendas e nos seus arredores, obteve-se um total de 123 espécies registradas localmente.
\end{abstract}

Palavras-chave: aves, trajeto irregular, Floresta Ombrófila Mista.

\section{Avifauna in Four Timber Farms in Irati, Paraná State: Analysis for Forest Certification}

\begin{abstract}
This study compared the community and diversity of birds in Mixed Ombrophylous Forest fragments of different sizes. To this end, eight fragments divided into sets forming two classes (from 20 to 70 ha and 100 to 420 ha) were selected in four farms in Guamirim district, municipality of Irati, Paraná state. The sampling method consisted of irregular transects traversed by natural areas. The experiment was conducted between 2009 and 2010, always in spring and autumn. Relative Frequency and Diversity were calculated by the Shannon index. The analyses Multiple Response Permutation Procedure (MRPP), Cluster, and MannWhitney (Wilcoxon) W test were used to compare the fragments. The results show that larger fragments present a distinct and more diverse community than patches smaller than 100 ha. Using irregular transects, we recorded 88 species of the total of 123 species recorded on site in transects and eventual observations.
\end{abstract}

Keywords: birds, irregular transects, Mixed Ombrophylous Forest. 


\section{INTRODUÇÃO E OBJETIVOS}

A intensa devastação das florestas tropicais projetou um cenário preocupante, principalmente devido à perda da biodiversidade. A fragmentação e os diferentes estágios de regeneração dos remanescentes destes ecossistemas são um desafio, tanto para a construção de medidas de conservação e manejo eficientes quanto para a gestão de restauração bem sucedida (Laurance, 2007).

A Floresta Atlântica Brasileira não é exceção. Restando menos de $20 \%$ de sua cobertura original, atualmente resume-se a um mosaico de remanescentes secundários, em diferentes fases sucessionais, predominantemente menores do que 100 ha (Ribeiro et al., 2009).

No Estado do Paraná, a Floresta Ombrófila Mista ocupava originalmente cerca de $37 \%$ de sua superfície, mas hoje se encontra extremamente fragmentada e reduzida a apenas $0,8 \%$ de florestas em estágio avançado de sucessão, sendo as florestas primárias praticamente inexistentes (Castella \& Britez, 2004). Tal situação se configura em um cenário de ameaça de desaparecimento deste ecossistema.

Há uma demanda de proprietários rurais para os levantamentos de fauna e flora de suas Reservas Legais para que possam submeter um plano de manejo ao órgão ambiental responsável ou para que obtenham a certificação ambiental/florestal de seus produtos, agregando, assim, maior valor de mercado na venda dos mesmos.

Paralelamente, a humanidade vem, cada vez mais, exigindo o cumprimento da legislação ambiental de modo a conservar nossos recursos naturais renováveis. Os especialistas há muito vêm alertando para a necessidade de se promover o uso racional dos recursos naturais, bem como a proteção de nossa fauna, flora e ecossistemas, principalmente os florestais.

A obtenção de documentos técnicos que apresentam diagnósticos de fauna, flora $\mathrm{e}$ ecossistemas das Reservas Legais (RLs) e Áreas de Preservação Permanente (APPs) é geralmente custosa para os proprietários.

Assim, a confecção desses documentos por universidades que contenham, em seu quadro de docentes e discentes, profissionais ligados às especialidades exigidas, proporciona vantagens à comunidade em termos de acesso facilitado aos conhecimentos acadêmico. Para a universidade, a grande vantagem é a possibilidade de oferecer aos acadêmicos a chance de aplicarem conhecimentos adquiridos durante o curso, além de uma experiência com o mercado de trabalho, empresas e comunidades envolvidas no assunto, exercendo o aprendizado (aplicar conhecimentos teóricos), a pesquisa (levantamentos de fauna e flora) e a extensão, em forma de prestação de serviço (geração de um documento técnico). A universidade ganha também em parcerias com empresas, equipamentos e materiais doados pelas mesmas ou por proprietários rurais, e na possibilidade de se inteirar melhor das necessidades da população envolvida.

Este trabalho refere-se ao levantamento quantitativo das espécies de aves das quatro fazendas da empresa Emílio B. Gomes \& Filhos S/A. As espécies constantes aqui são as registradas em áreas de floresta, em diversos estágios de regeneração, e outros ecossistemas, como campos, culturas, brejos e plantios florestais.

Nesta avaliação, a intenção é verificar quais são os locais mais importantes biológica e ecologicamente, para fins de auditoria e certificação florestal. A empresa certificadora responsável pela auditoria foi a Forest Stewardship Council (FSC).

Neste contexto, o presente estudo pretende responder às seguintes perguntas:

- Existem diferenças na frequência relativa de aves em fragmentos com diferentes tamanhos?

- A diversidade da comunidade de aves dos fragmentos é distinta?

\section{MATERIAL E MÉTODOS}

\subsection{Coleta de dados}

$\mathrm{O}$ método de amostragem consistiu em trajetos irregulares percorridos pelas áreas naturais (Reservas Legais e Áreas de Preservação Permanente) das fazendas da empresa Emílio B. Gomes \& Filhos S/A, no distrito de Guamirim, município Irati, Estado do Paraná, Brasil. A localização dos fragmentos florestais estudados é representada na Figura 1. Durante os 
percursos, todos os indivíduos que puderam ser seguramente identificados foram registrados para cada espécie, desde que avistado dentro das áreas naturais protegidas (RLs e APPs), sendo o tempo (em horas) contado como unidade amostral. Procurou-se adequar o esforço amostral, em horas, ao tamanho de cada fragmento. A vegetação nativa original na área de estudo é a Floresta Ombrófila Mista Montana.

As análises foram divididas em quatro, já que se consideram quatro fazendas distintas no local. Em cada fazenda, foram realizados quatro trajetos, sendo dois em cada fragmento. Em cada fazenda, foi possível distinguir dois fragmentos, constituídos de Reserva Legal e área contínua a esta, classificada como Área de Preservação Permanente, num total de oito fragmentos. Os levantamentos foram realizados entre maio de 2009 e setembro de 2010, sempre nas estações primavera (abril e maio) e outono (setembro, outubro e novembro), consideradas mais adequadas para levantamentos de aves por observação, quando não é possível fazer campanhas mensais.

Para fins de comparação entre os fragmentos, foi calculada a Frequência Relativa (FR) de cada espécie para cada um dos fragmentos. A FR é dada pelo número de indivíduos $(\mathrm{Ni})$ registrados na espécie $i$ em relação ao número de horas de observação.

Além do levantamento quantitativo, foram realizadas algumas visitas nas fazendas e seus arredores, visando a registrar espécies ocasionalmente não captadas pelo método dos trajetos irregulares, $\mathrm{o}$ que resultou em uma lista mais completa das aves locais.

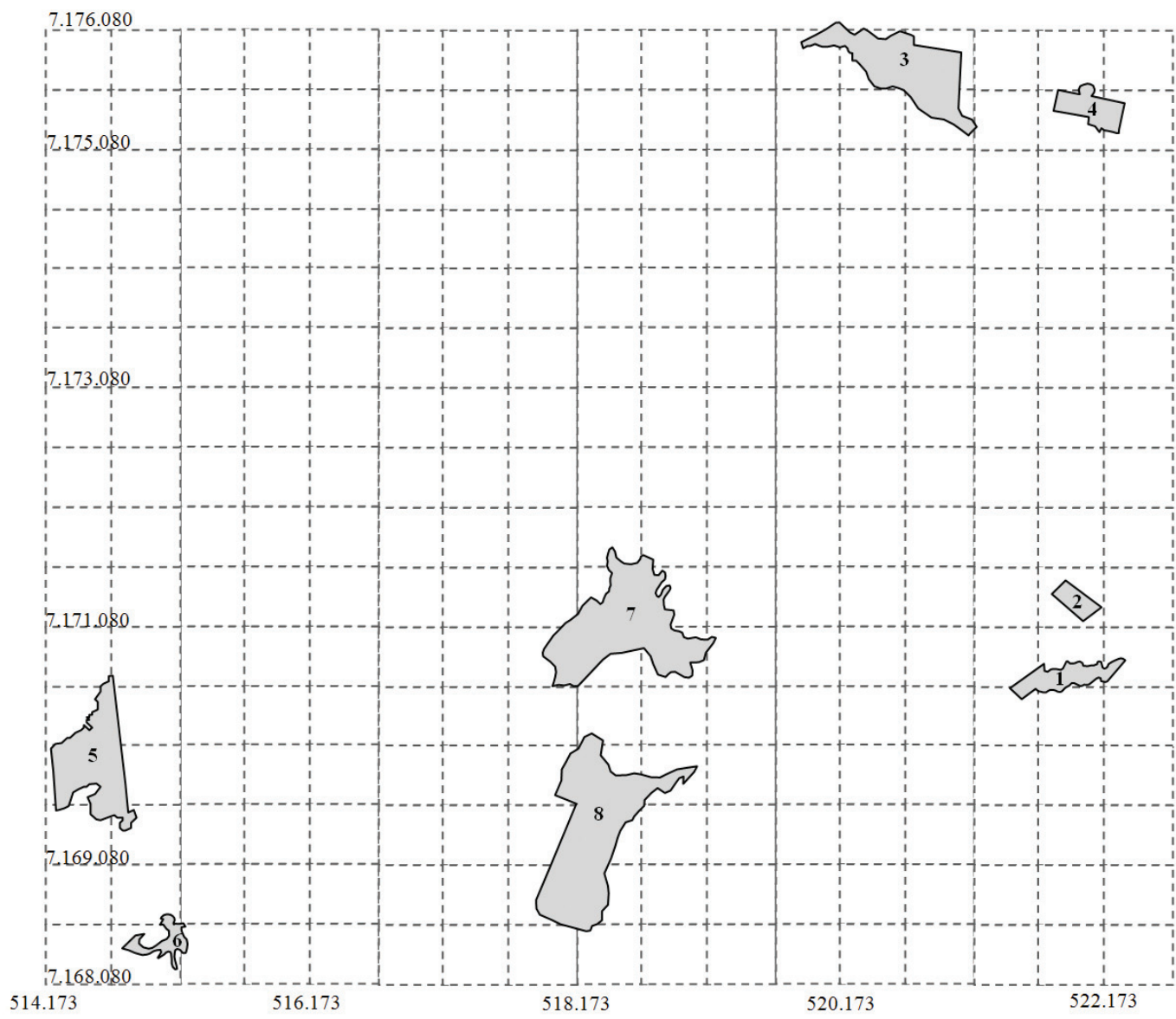

Figura 1. Localização da área de estudo em Irati, Paraná. Fragmentos estão numerados de um a oito. Autor: Deise Regina Lazzarotto.

Figure 1. Localization of the focused area in Irati, Paraná. Patches are numbered from one to eight. Author: Deise Regina Lazzarotto. 


\subsection{Análise de dados}

Para comparar a diversidade (Índice de Shannon) entre os conjuntos de fragmentos - que somados vão de 20 até 70 ha e de 100 até 420 ha -, o teste MannWhitney (Wilcoxon) W a 5\% de significância foi utilizado (Zar, 1999).

A matriz de Frequência Relativa (FR) foi utilizada para a construção de um cluster (análise de agrupamento hierárquico), sendo a distância calculada por Jaccard, e utilizado o método de ligação pelo vizinho mais distante (Farthest Neighbor), conforme proposto por McCune \& Grace (2002). O teste multivariado para comparação entre grupos Multiple Response Permutation Procedure (MRPP) foi usado para averiguar se existe diferença entre a frequência relativa de aves nos fragmentos maiores, formados por quatro repetições, e menores, também formados por quatro repetições. A distância utilizada foi a mesma do método de classificação hierárquico (Jaccard). O número de permutações foi igual a 999 $(a=0,05)$.

As análises foram calculadas com o auxílio do programa PC-ORD versão 5.0 (McCune \& Grace, 2002).

Os dados qualitativos foram discutidos em termos de conservação de algumas espécies, que demandam mais atenção, e da comunidade de aves locais como um todo.

\section{RESULTADOS E DISCUSSÃO}

\subsection{Riqueza e diversidade}

Foram registrados 603 indivíduos, distribuídos em 88 espécies de aves, durante os trajetos amostrais nas florestas nativas das quatro fazendas: Fazenda Arroio Grande I, Fazenda Arroio Grande III, Fazenda Água Quente I e Fazenda Água Quente II (dividida em Área A e Área B).

A Tabela 1 mostra o número de espécies de aves encontradas em cada fazenda e o tamanho de suas Reservas Legais somadas às suas Áreas de Preservação Permanente, as quais constituem as áreas naturais contínuas amostradas em cada uma. Os dados de tamanho de áreas protegidas foram fornecidos pela própria empresa, com base em mapeamento realizado em 2006 . Os dados de riqueza e diversidade para cada fragmento são apresentados na Tabela 2.

Os dois grandes fragmentos e suas áreas adjacentes da Fazenda Água Quente I possuem um valor de conservação superior aos demais, indicado pela maior riqueza (59 espécies, Tabela 1) e diversidade (Índice de Shannon: fragmento $8=3,85$ e fragmento $7=3,64)$. O conjunto de fragmentos de 20 a 70 ha apresentou diversidade menor do que o conjunto de 100 a 420 ha $(\mathrm{p}<0,05 \%$ para o teste Mann-Whitney W). Tal fato pode ser explicado pelas seguintes características encontradas:

- Tamanho maior, em relação aos outros fragmentos, o que significa que possui também maior núcleo florestal, isto é, uma área no centro do fragmento livre de efeito de borda, com maior possibilidade de abrigar espécies exigentes em termos de habitat. Normalmente, estas espécies estão entre as mais raras e endêmicas, já que seu habitat, a Floresta Ombrófila Mista, está extremamente ameaçada no Estado e mesmo no País. Tais fragmentos possuem cerca de 66 ha e 58 ha e a área total, somando-se a RL e a APP da fazenda, é de 419,02 ha;

Tabela 1. Riqueza de aves e tamanho das áreas naturais protegidas (Reserva Legal + Área de Preservação Permanente) em cada fazenda.

Table 1. Richness of birds and size of protected natural areas (Legal Reserve + Permanent Preservation Area) on each farm.

\begin{tabular}{|cccc|}
\hline Fazenda & $\begin{array}{c}\text { Número dos fragmentos } \\
\text { amostrados }\end{array}$ & $\begin{array}{c}\text { Número de espécies de } \\
\text { aves registradas (riqueza) }\end{array}$ & $\begin{array}{c}\text { Área de mata nativa } \\
\text { (em ha) }\end{array}$ \\
\hline Arroio Grande I & 3 e 4 & 31 & 108,97 \\
\hline Arroio Grande III & 1 e 2 & 31 & 22,03 \\
\hline Água Quente I & 7 e 8 & 59 & 419,02 \\
\hline Água Quente II - Áreas A+B & 5 e 6 & 44 & 56,9 \\
\hline TOTAL & 8 fragmentos & 88 & 606,92 \\
\hline
\end{tabular}


Tabela 2. Riqueza e diversidade de aves nos fragmentos florestais das fazendas de Guamirim, Irati-PR.

Table 2. Richness and diversity of birds in forest fragments of Guamirim, Irati, PR farms.

\begin{tabular}{ccc|}
\hline Fragmentos & Riqueza & Shannon $(\mathbf{H})$ \\
\hline 8 & 47 & 3,850147 \\
7 & 38 & 3,637586 \\
\hline 6 & 29 & 3,367296 \\
\hline 5 & 26 & 3,258096 \\
\hline 4 & 22 & 3,091043 \\
\hline 3 & 16 & 2,772589 \\
\hline 2 & 21 & 3,044523 \\
\hline 1 & 25 & 3,218876 \\
\hline
\end{tabular}

- O mesmo pode-se dizer em relação ao fator 'forma e núcleo florestal', já que esses fragmentos não são tão alongados quanto a maioria dos demais;

- Melhor estado de conservação da vegetação original, indicado pelo inventário arbóreo (Relatório Técnico não publicado). Talvez esta seja a principal característica a influir na avifauna, sendo, portanto, aconselhados maiores estudos neste sentido;

- Há corredores ecológicos, em forma de vegetação ripária, ligando os dois grandes fragmentos a outros menores e a fragmentos em propriedades vizinhas.

Além do levantamento quantitativo, exclusivamente nos fragmentos florestais, foram anotadas as espécies vistas ao acaso em qualquer área dentro das fazendas e nos seus arredores. Assim, foram registradas outras 28 espécies nas fazendas, totalizando 116 espécies de aves no conjunto de propriedades da empresa. Outras sete espécies foram registradas exclusivamente em áreas ao redor das fazendas, totalizando 123 espécies locais. Todas as espécies registradas localmente estão listadas na Tabela 3, pela ordem sugerida pelo Comitê Brasileiro de Registros Ornitológicos (CBRO, 2011).

Há uma observação não confirmada de Molothrus rufoaxillaris Cassin, 1866 (vira-bostapicumã), na Fazenda Água Quente I, em 2009. Um único indivíduo encontrava-se em área com predominância de matagal e outros ambientes abertos/semiabertos heterogêneos.

Alguns trabalhos publicados permitem comparar a riqueza de aves registradas na região, nos municípios de Irati e Fernandes Pinheiro. No município de Fernandes Pinheiro, Romaniuk et al. (2011) realizaram levantamento preliminar de aves na Floresta Nacional (FLONA) de Irati, por meio de rede-neblina (176 horas-rede), e encontraram 19 espécies, distribuídas em 11 famílias. Posteriormente, em um levantamento complementar, totalizando então 376 horas-rede (Romaniuk, 2011), foram registradas 22 espécies em 14 famílias. No mesmo município, Winagraski et al. (2011) registraram 18 espécies, em 11 famílias, na Estação Ecológica de Fernandes Pinheiro, usando o mesmo método (104 horas-rede).

Em 1990, foram registradas 114 espécies de aves na FLONA de Irati (FUPEF, 1990), através de observação, em diversos ambientes visitados. Volpato (2009) e Volpato et al. (2010) também registraram no local 114 espécies de aves 19 anos depois, em um levantamento com maior esforço amostral por observação, sendo feita amostragem em floresta nativa, floresta de araucária plantada em regeneração e talhão de pinus com regeneração de sub-bosque; destas espécies, 48 foram classificadas como espécies florestais-dependentes e 66 como espécies florestais-generalistas. Entre março de 2007 e junho de 2010, Marcelino \& Romaniuk (2010) realizaram observações ocasionais na FLONA de Irati, percorrendo todos os ambientes e encontrando 81 espécies de aves.

No Colégio Florestal Presidente Costa e Silva, em Irati, no bairro Vila São João, Marcelino \& Adenesky-Filho (2008) encontraram 41 espécies de aves distribuídas em 20 famílias. No Campus de Irati da Universidade Estadual do CentroOeste - UNICENTRO, no bairro Riozinho, foram registradas 58 espécies de aves, distribuídas em 28 famílias (Marcelino \& Silva, 2008).

\subsection{Frequência}

A Frequência Relativa de cada espécie para cada fragmento é mostrada na Tabela 4. De modo geral, as espécies mais frequentes e abundantes nos fragmentos foram: o pombão (Patagioenas picazuro), a gralha-picaça (Cyanocorax chrysops), o tico-tico (Zonotrichia capensis), o pula-pula (Basileuterus culicivorus) e o pula-pula-assobiador (Basileuterus leucoblepharus). O tico-tico é uma espécie típica de campo e, quando presente em florestas, indica sua 
Tabela 3. Aves registradas em Guamirim, Irati-PR. $\left(^{*}\right)=$ espécies não registradas durante os trajetos irregulares. Em negrito $=$ espécies registradas dentro das fazendas.

Table 3. Birds recorded in Guamirim, Irati, PR. $\left({ }^{*}\right)=$ Species that were not recorded during the irregular transects. Bold $=$ Species recorded in the farms.

\section{Nome do Táxon}

Crypturellus obsoletus (Temminck, 1815)

Rhynchotus rufescens (Temminck, 1815)

Amazonetta brasiliensis (Gmelin, 1789)

Penelope obscura Temminck, 1815

Bubulcus ibis (Linnaeus, 1758)

Phimosus infuscatus (Lichtenstein, 1823)

Theristicus caudatus (Boddaert, 1783)

Cathartes aura (Linnaeus, 1758)

Coragyps atratus (Bechstein, 1793)

Chondrohierax uncinatus (Temminck, 1822)

Accipiter poliogaster (Temminck, 1824)

Accipiter striatus Vieillot, 1808

Rupornis magnirostris (Gmelin, 1788)

Caracara plancus (Miller, 1777)

Milvago chimachima (Vieillot, 1816)

Aramides saracura (Spix, 1825)

Gallinula galeata (Lichtenstein,1818)

Vanellus chilensis (Molina, 1782)

Jacana jacana (Linnaeus, 1766)

Columbina talpacoti (Temminck, 1811)

Columbina squammata (Lesson, 1831)

Columba livia Gmelin, 1789

Patagioenas picazuro (Temminck, 1813)

Patagioenas cayennensis (Bonnaterre, 1792)

Zenaida auriculata (Des Murs, 1847)

Leptotila verreauxi Bonaparte, 1855

Leptotila rufaxilla (Richard \& Bernard, 1792)

Pyrrhura frontalis (Vieillot, 1817)

Pionus maximiliani (Kuhl, 1820)

Piaya cayana (Linnaeus, 1766)

Tapera naevia (Linnaeus, 1766)

Stephanoxis lalandi (Vieillot, 1818)

Chlorostilbon lucidus (Shaw, 1812)

Leucochloris albicollis (Vieillot, 1818)

Amazilia versicolor (Vieillot, 1818)

Trogon surrucura Vieillot, 1817

Megaceryle torquata (Linnaeus, 1766)

Chloroceryle amazona (Latham, 1790)

Ramphastos dicolorus Linnaeus, 1766

Picumnus temminckii Lafresnaye, 1845

Melanerpes candidus (Otto, 1796)

Veniliornis spilogaster (Wagler, 1827)

Colaptes melanochloros (Gmelin, 1788)

Colaptes campestris (Vieillot, 1818)

Dryocopus lineatus (Linnaeus, 1766)

Dysithamnus mentalis (Temminck, 1823)
Nome Popular

inhambuguaçu

perdiz *

pé-vermelho*

jacuaçu $^{*}$

garça-vaqueira $^{\star}$

tapicuru-de-cara-pelada ${ }^{\star}$

curicaca

urubu-de-cabeça-vermelha*

urubu-de-cabeça-preta ${ }^{*}$

caracoleiro

tauató-pintado

gavião-miúdo*

gavião-carijó

caracará

carrapateiro

saracura-do-mato

frango-d'água-comum ${ }^{*}$

quero-quero*

jaçanã

rolinha-roxa

fogo-apagou*

pombo-doméstico pombão

pomba-galega ${ }^{*}$

pomba-de-bando*

juriti-pupu

juriti-gemedeira

tiriba-de-testa-vermelha

maitaca-verde*

alma-de-gato*

saci

beija-flor-de-topete

besourinho-de-bico-vermelho

beija-flor-de-papo-branco

beija-flor-de-banda-branca*

surucuá-variado

martim-pescador-grande ${ }^{\star}$

martim-pescador-verde*

tucano-de-bico-verde

pica-pau-anão-de-coleira pica-pau-branco

picapauzinho-verde-carijó

pica-pau-verde-barrado*

pica-pau-do-campo

pica-pau-de-banda-branca choquinha-lisa 
Tabela 3. Continuação

Table 3. Continued...

\begin{tabular}{|c|c|}
\hline Nome do Táxon & Nome Popular \\
\hline Thamnophilus caerulescens Vieillot, 1816 & choca-da-mata \\
\hline Drymophila malura (Temminck, 1825) & choquinha-carijó \\
\hline Chamaeza campanisona (Lichtenstein, 1823) & tovaca-campainha \\
\hline Sclerurus scansor (Ménétriès, 1835) & vira-folha ${ }^{*}$ \\
\hline Sittasomus griseicapillus (Vieillot, 1818) & arapaçu-verde \\
\hline Lepidocolaptes falcinellus (Cabanis \& Heine, 1859) & arapaçu-escamado-do-sul \\
\hline Dendrocolaptes platyrostris Spix, 1825 & arapaçu-grande \\
\hline Xenops rutilans Temminck, 1821 & bico-virado-carijó \\
\hline Furnarius rufus (Gmelin, 1788) & joão-de-barro \\
\hline Philydor rufum (Vieillot, 1818) & limpa-folha-de-testa-baia \\
\hline Anabacerthia amaurotis (Temminck, 1823) & limpa-folha-miúdo \\
\hline Syndactyla rufosuperciliata (Lafresnaye, 1832) & trepador-quiete \\
\hline Leptasthenura striolata (Pelzeln, 1856) & grimpeirinho \\
\hline Synallaxis ruficapilla Vieillot, 1819 & pichororé \\
\hline Synallaxis cinerascens Temminck, 1823 & pi-puí \\
\hline Synallaxis spixi Sclater, 1856 & joão-teneném \\
\hline Chiroxiphia caudata (Shaw \& Nodder, 1793) & tangará \\
\hline Mionectes rufiventris Cabanis, 1846 & abre-asa-de-cabeça-cinza \\
\hline Leptopogon amaurocephalus Tschudi, 1846 & cabeçudo \\
\hline Phylloscartes ventralis (Temminck, 1824) & borboletinha-do-mato \\
\hline Tolmomyias sulphurescens (Spix, 1825) & bico-chato-de-orelha-preta \\
\hline Poecilotriccus plumbeiceps (Lafresnaye, 1846) & tororó \\
\hline Camptostoma obsoletum (Temminck, 1824) & risadinha \\
\hline Elaenia flavogaster (Thunberg, 1822) & guaracava-de-barriga-amarela \\
\hline Phyllomyias fasciatus (Thunberg, 1822) & piolhinho \\
\hline Attila phoenicurus Pelzeln, 1868 & capitão-castanho \\
\hline Pitangus sulphuratus (Linnaeus, 1766) & bem-te-vi \\
\hline Myiodynastes maculatus (Statius Muller, 1776) & bem-te-vi-rajado \\
\hline Megarynchus pitangua (Linnaeus, 1766) & neinei \\
\hline Tyrannus melancholicus Vieillot, 1819 & suiriri \\
\hline Tyrannus savana Vieillot, 1808 & tesourinha* \\
\hline Colonia colonus (Vieillot, 1818) & viuvinha \\
\hline Myiophobus fasciatus (Statius Muller, 1776) & filipe \\
\hline Satrapa icterophrys (Vieillot, 1818) & suiriri-pequeno* $^{*}$ \\
\hline Muscipipra vetula (Lichtenstein, 1823) & tesoura-cinzenta* \\
\hline Cyclarhis gujanensis (Gmelin, 1789) & pitiguari \\
\hline Vireo olivaceus (Linnaeus, 1766) & juruviara \\
\hline Cyanocorax chrysops (Vieillot, 1818) & gralha-picaça \\
\hline Pygochelidon cyanoleuca (Vieillot, 1817) & andorinha-pequena-de-casa ${ }^{*}$ \\
\hline Stelgidopteryx ruficollis (Vieillot, 1817) & andorinha-serradora* \\
\hline Progne chalybea (Gmelin, 1789) & andorinha-doméstica-grande ${ }^{\star}$ \\
\hline Troglodytes musculus Naumann, 1823 & corruíra \\
\hline Turdus rufiventris Vieillot, 1818 & sabiá-laranjeira \\
\hline Turdus leucomelas Vieillot, 1818 & sabiá-barranco \\
\hline Turdus amaurochalinus Cabanis, 1850 & sabiá-poca \\
\hline Turdus subalaris (Seebohm, 1887) & sabiá-ferreiro \\
\hline Turdus albicollis Vieillot, 1818 & sabiá-coleira \\
\hline Coereba flaveola (Linnaeus, 1758) & cambacica \\
\hline
\end{tabular}


Tabela 3. Continuação

Table 3. Continued...

\section{Nome do Táxon}

Saltator similis d'Orbigny \& Lafresnaye, 1837

Pyrrhocoma ruficeps (Strickland, 1844)

Tachyphonus coronatus (Vieillot, 1822)

Lanio melanops (Vieillot, 1818)

Tangara sayaca (Linnaeus, 1766)

Tangara preciosa (Cabanis, 1850)

Stephanophorus diadematus (Temminck, 1823)

Pipraeidea bonariensis (Gmelin, 1789)

Conirostrum speciosum (Temminck, 1824)

Zonotrichia capensis (Statius Muller, 1776)

Ammodramus humeralis (Bosc, 1792)

Poospiza lateralis (Nordmann, 1835)

Sicalis flaveola (Linnaeus, 1766)

Volatinia jacarina (Linnaeus, 1766)

Sporophila caerulescens (Vieillot, 1823)

Cyanoloxia glaucocaerulea (d'Orbigny \& Lafresnaye, 1837)

Cyanoloxia brissonii (Lichtenstein, 1823)

Parula pitiayumi (Vieillot, 1817)

Basileuterus culicivorus (Deppe, 1830)

Basileuterus leucoblepharus (Vieillot, 1817)

Cacicus chrysopterus (Vigors, 1825)

Icterus cayanensis (Linnaeus, 1766)

Gnorimopsar chopi (Vieillot, 1819)

Sporagra magellanica (Vieillot, 1805)

\section{Nome Popular}

trinca-ferro-verdadeiro

cabecinha-castanha

tiê-preto

tiê-de-topete

sanhaçu-cinzento

saíra-preciosa

sanhaçu-frade

sanhaçu-papa-laranja

figuinha-de-rabo-castanho tico-tico

tico-tico-do-campo* quete

canário-da-terra-verdadeiro* tiziu $^{*}$

coleirinho* $^{*}$

azulinho

azulão

mariquita

pula-pula

pula-pula-assobiador

tecelão

inhapim

graúna $^{*}$

pintassilgo* $^{*}$ degradação ou grande quantidade de bordas em fragmentos. O pombão é uma espécie generalista, ocorrendo tanto em áreas abertas (naturais ou antrópicas) quanto em florestas, não sendo, portanto, uma espécie bioindicadora. Já as demais com alta frequência são espécies que ocorrem em núcleo e borda de florestas.

A análise de agrupamento hierárquico - cluster - (Figura 2) apontou grupos distintos em função do tamanho do fragmento. O teste multivariado MRPP ratificou esta informação, separando estatisticamente a composição de aves dos fragmentos maiores dos menores $(p>0,05)$.

\subsection{Conservação}

Foram registradas quatro espécies constantes no Livro Vermelho da Fauna Ameaçada no Estado do Paraná (Mikich \& Bérnils, 2004): Chondrohierax uncinatus (caracoleiro, Ordem Falconiformes,
Família Accipitridae, registrado um indivíduo no Fragmento 3, Fazenda Arroio Grande I) na Categoria proposta VU (Vulnerável - Vulnerable - espécie que, de acordo com os critérios específicos, está sob um risco alto de extinção na natureza); Accipiter poliogaster (tauató-pintado, Ordem Falconiformes, Família Accipitridae, registrado um indivíduo no Fragmento 8, Fazenda Água Quente I) na Categoria proposta DD (Dados Insuficientes - Data Deficient - espécie que necessita de mais dados, principalmente de abundância e distribuição, para que seu status possa ser corretamente avaliado); Leptasthenura striolata (grimpeirinho, Ordem Passeriformes, Família Furnariidae, registrados três indivíduos no Fragmento 8, Fazenda Água Quente I) na Categoria proposta DD; Passerina (Cyanoloxia) glaucocaerulea (azulinho, Ordem Passeriformes, Família Emberizidae, registrados dois indivíduos no Fragmento 8, Fazenda Água Quente I) na Categoria proposta NT (Quase Ameaçada - Near 
Tabela 4. Frequência Relativa das espécies de aves em fragmentos de Guamirim, Irati-PR.

Table 4. Relative Frequency of birds in fragments of Guamirim, Irati, PR.

\begin{tabular}{|c|c|c|c|c|c|c|c|c|}
\hline Nome do Táxon & $\begin{array}{c}\text { Frag } \\
8\end{array}$ & $\begin{array}{c}\text { Frag } \\
7\end{array}$ & $\begin{array}{c}\text { Frag } \\
6\end{array}$ & $\begin{array}{c}\text { Frag } \\
5\end{array}$ & $\begin{array}{c}\text { Frag } \\
4\end{array}$ & $\begin{array}{c}\text { Frag } \\
3\end{array}$ & $\begin{array}{c}\text { Frag } \\
2\end{array}$ & $\begin{array}{c}\text { Frag } \\
1\end{array}$ \\
\hline Crypturellus obsoletus (Temminck, 1815) & 0,20 & 0,00 & 0,83 & 0,89 & 0,00 & 0,00 & 0,54 & 0,00 \\
\hline Theristicus caudatus (Boddaert, 1783) & 0,00 & 0,00 & 0,41 & 0,00 & 0,00 & 0,00 & 0,00 & 0,00 \\
\hline Chondrohierax uncinatus (Temminck, 1822) & 0,00 & 0,00 & 0,00 & 0,00 & 0,00 & 0,48 & 0,00 & 0,00 \\
\hline Accipiter poliogaster (Temminck, 1824) & 0,20 & 0,00 & 0,00 & 0,00 & 0,00 & 0,00 & 0,00 & 0,00 \\
\hline Rupornis magnirostris (Gmelin, 1788) & 0,20 & 0,00 & 0,83 & 0,00 & 0,00 & 0,00 & 0,00 & 0,39 \\
\hline Caracara plancus (Miller, 1777) & 0,00 & 0,00 & 0,00 & 0,89 & 0,00 & 0,00 & 0,00 & 0,00 \\
\hline Milvago chimachima (Vieillot, 1816) & 0,00 & 0,86 & 0,41 & 0,00 & 0,00 & 0,00 & 0,00 & 0,00 \\
\hline Aramides saracura (Spix, 1825) & 0,00 & 0,57 & 0,00 & 0,00 & 1,33 & 0,00 & 0,00 & 0,00 \\
\hline Columbina talpacoti (Temminck, 1811) & 0,00 & 0,00 & 0,00 & 0,00 & 0,00 & 0,00 & 0,00 & 1,55 \\
\hline Patagioenas picazuro (Temminck, 1813) & 0,81 & 0,86 & 0,41 & 0,00 & 2,00 & 0,96 & 1,09 & 2,71 \\
\hline Leptotila verreauxi Bonaparte, 1855 & 0,41 & 0,57 & 2,48 & 0,44 & 0,00 & 0,96 & 1,09 & 1,16 \\
\hline Leptotila rufaxilla (Richard \& Bernard, 1792) & 0,00 & 0,00 & 0,00 & 0,00 & 0,00 & 0,96 & 0,54 & 0,78 \\
\hline Pyrrhura frontalis (Vieillot, 1817) & 0,41 & 0,00 & 0,00 & 0,00 & 0,00 & 0,00 & 0,00 & 0,00 \\
\hline Tapera naevia (Linnaeus, 1766) & 0,20 & 0,00 & 0,00 & 0,00 & 0,00 & 0,00 & 0,00 & 0,00 \\
\hline Stephanoxis lalandi (Vieillot, 1818) & 0,00 & 0,00 & 0,00 & 0,00 & 0,00 & 0,00 & 2,17 & 0,00 \\
\hline Chlorostilbon lucidus (Shaw, 1812) & 0,41 & 0,00 & 0,00 & 0,00 & 0,00 & 0,00 & 0,00 & 0,00 \\
\hline Leucochloris albicollis (Vieillot, 1818) & 0,20 & 0,29 & 0,00 & 0,00 & 0,67 & 0,00 & 0,00 & 0,00 \\
\hline Trogon surrucura Vieillot, 1817 & 0,20 & 1,71 & 0,00 & 0,44 & 0,00 & 0,48 & 0,00 & 0,39 \\
\hline Ramphastos dicolorus Linnaeus, 1766 & 0,00 & 0,00 & 0,83 & 0,89 & 0,00 & 0,00 & 0,00 & 0,00 \\
\hline Picumnus temminckii Lafresnaye, 1845 & 0,00 & 0,29 & 0,41 & 0,00 & 0,67 & 0,00 & 0,00 & 0,00 \\
\hline Melanerpes candidus (Otto, 1796) & 0,61 & 0,00 & 0,00 & 0,00 & 0,00 & 0,00 & 0,00 & 0,00 \\
\hline Veniliornis spilogaster (Wagler, 1827) & 0,20 & 0,86 & 0,41 & 0,00 & 0,00 & 0,00 & 0,00 & 0,00 \\
\hline Colaptes campestris (Vieillot, 1818) & 0,00 & 0,00 & 0,00 & 0,00 & 0,00 & 0,00 & 0,00 & 0,39 \\
\hline Dryocopus lineatus (Linnaeus, 1766) & 0,00 & 0,00 & 0,00 & 0,00 & 0,00 & 0,00 & 0,00 & 0,39 \\
\hline Dysithamnus mentalis (Temminck, 1823) & 0,00 & 0,29 & 0,83 & 1,78 & 0,67 & 0,96 & 0,54 & 0,00 \\
\hline Thamnophilus caerulescens Vieillot, 1816 & 0,00 & 0,00 & 0,00 & 1,78 & 0,67 & 0,00 & 0,00 & 0,00 \\
\hline Drymophila malura (Temminck, 1825) & 0,00 & 0,57 & 0,00 & 0,00 & 0,00 & 0,00 & 0,00 & 0,00 \\
\hline Chamaeza campanisona (Lichtenstein, 1823) & 0,00 & 0,00 & 0,00 & 0,00 & 0,67 & 0,00 & 0,00 & 0,00 \\
\hline Sittasomus griseicapillus (Vieillot, 1818) & 0,81 & 1,43 & 0,83 & 1,78 & 0,67 & 0,00 & 0,00 & 1,16 \\
\hline Lepidocolaptes falcinellus (Cabanis \& Heine, 1859) & 0,41 & 0,00 & 0,00 & 0,00 & 0,00 & 0,00 & 0,00 & 0,00 \\
\hline Dendrocolaptes platyrostris Spix, 1825 & 0,20 & 0,00 & 0,00 & 0,44 & 0,00 & 0,00 & 1,09 & 0,00 \\
\hline Xenops rutilans Temminck, 1821 & 0,41 & 0,00 & 0,41 & 0,00 & 1,33 & 0,00 & 0,00 & 0,00 \\
\hline Furnarius rufus (Gmelin, 1788) & 0,00 & 0,29 & 0,41 & 0,00 & 0,00 & 0,00 & 0,00 & 0,00 \\
\hline Philydor rufum (Vieillot, 1818) & 0,00 & 0,00 & 0,00 & 0,44 & 0,00 & 0,00 & 0,00 & 0,00 \\
\hline Anabacerthia amaurotis (Temminck, 1823) & 0,20 & 0,00 & 0,00 & 0,00 & 0,00 & 0,00 & 0,00 & 0,39 \\
\hline Syndactyla rufosuperciliata (Lafresnaye, 1832) & 0,00 & 0,00 & 0,00 & 0,00 & 0,00 & 0,48 & 0,00 & 0,00 \\
\hline Leptasthenura striolata (Pelzeln, 1856) & 0,61 & 0,00 & 0,00 & 0,00 & 0,00 & 0,00 & 0,00 & 0,00 \\
\hline Synallaxis ruficapilla Vieillot, 1819 & 0,00 & 0,00 & 0,00 & 0,00 & 0,67 & 0,00 & 0,00 & 0,00 \\
\hline Synallaxis cinerascens Temminck, 1823 & 1,43 & 0,57 & 0,00 & 0,89 & 0,00 & 0,00 & 1,09 & 0,78 \\
\hline Synallaxis spixi Sclater, 1856 & 1,22 & 0,00 & 0,00 & 0,44 & 1,33 & 0,96 & 0,54 & 1,94 \\
\hline Chiroxiphia caudata (Shaw \& Nodder, 1793) & 0,00 & 0,00 & 0,41 & 0,00 & 0,00 & 0,00 & 0,00 & 0,00 \\
\hline Mionectes rufiventris Cabanis, 1846 & 0,61 & 0,00 & 0,00 & 0,00 & 0,00 & 0,00 & 0,00 & 0,00 \\
\hline Leptopogon amaurocephalus Tschudi, 1846 & 0,41 & 0,00 & 0,00 & 0,44 & 0,00 & 0,00 & 0,00 & 0,00 \\
\hline Phylloscartes ventralis (Temminck, 1824) & 0,00 & 0,00 & 0,00 & 0,00 & 0,67 & 0,00 & 0,00 & 0,00 \\
\hline Tolmomyias sulphurescens (Spix, 1825) & 0,61 & 0,29 & 1,24 & 1,33 & 1,33 & 0,00 & 0,54 & 0,39 \\
\hline Poecilotriccus plumbeiceps (Lafresnaye, 1846) & 1,43 & 0,29 & 0,00 & 0,89 & 0,00 & 0,00 & 0,54 & 0,78 \\
\hline Camptostoma obsoletum (Temminck, 1824) & 0,41 & 0,29 & 0,41 & 0,00 & 0,67 & 0,00 & 0,00 & 0,78 \\
\hline
\end{tabular}


Tabela 4. Continuação

Table 4. Continued...

\begin{tabular}{|c|c|c|c|c|c|c|c|c|}
\hline Nome do Táxon & $\begin{array}{c}\text { Frag } \\
8\end{array}$ & $\begin{array}{c}\text { Frag } \\
7\end{array}$ & $\begin{array}{c}\text { Frag } \\
6\end{array}$ & $\begin{array}{c}\text { Frag } \\
5\end{array}$ & $\begin{array}{c}\text { Frag } \\
4\end{array}$ & $\begin{array}{c}\text { Frag } \\
3\end{array}$ & $\begin{array}{c}\text { Frag } \\
2\end{array}$ & $\begin{array}{c}\text { Frag } \\
1\end{array}$ \\
\hline Elaenia flavogaster (Thunberg, 1822) & 0,81 & 1,14 & 0,00 & 0,00 & 0,00 & 0,00 & 0,00 & 0,00 \\
\hline Phyllomyias fasciatus (Thunberg, 1822) & 0,00 & 0,00 & 0,00 & 0,00 & 0,67 & 0,00 & 0,00 & 0,00 \\
\hline Attila phoenicurus Pelzeln, 1868 & 0,81 & 0,00 & 0,00 & 0,00 & 0,00 & 0,00 & 0,00 & 0,00 \\
\hline Pitangus sulphuratus (Linnaeus, 1766) & 0,41 & 1,14 & 1,65 & 0,00 & 0,00 & 0,00 & 0,54 & 0,00 \\
\hline Myiodynastes maculatus (Statius Muller, 1776) & 1,63 & 3,14 & 0,00 & 0,00 & 0,00 & 0,00 & 0,00 & 0,00 \\
\hline Megarynchus pitangua (Linnaeus, 1766) & 0,41 & 0,29 & 0,41 & 0,00 & 0,00 & 0,00 & 0,00 & 0,00 \\
\hline Tyrannus melancholicus Vieillot, 1819 & 0,41 & 0,00 & 0,00 & 0,00 & 0,00 & 0,00 & 0,00 & 0,00 \\
\hline Colonia colonus (Vieillot, 1818) & 0,41 & 0,00 & 0,00 & 0,00 & 0,00 & 0,00 & 1,09 & 0,00 \\
\hline Cyclarhis gujanensis (Gmelin, 1789) & 1,43 & 0,57 & 0,00 & 0,89 & 1,33 & 0,96 & 0,54 & 0,39 \\
\hline Vireo olivaceus (Linnaeus, 1766) & 1,22 & 1,14 & 0,00 & 0,00 & 0,00 & 0,00 & 0,00 & 0,00 \\
\hline Cyanocorax chrysops (Vieillot, 1818) & 1,22 & 1,71 & 0,83 & 2,22 & 2,00 & 0,00 & 0,54 & 1,16 \\
\hline Troglodytes musculus Naumann, 1823 & 0,20 & 0,00 & 0,00 & 0,00 & 0,00 & 0,48 & 0,54 & 1,16 \\
\hline Turdus rufiventris Vieillot, 1818 & 1,02 & 0,57 & 0,41 & 1,33 & 0,67 & 0,00 & 0,00 & 0,00 \\
\hline Turdus leucomelas Vieillot, 1818 & 0,00 & 0,00 & 1,24 & 0,00 & 0,00 & 0,00 & 0,00 & 0,00 \\
\hline Turdus amaurochalinus Cabanis, 1850 & 0,20 & 0,57 & 0,00 & 0,00 & 0,00 & 0,00 & 0,00 & 0,00 \\
\hline Turdus subalaris (Seebohm, 1887) & 0,00 & 0,00 & 2,07 & 0,00 & 0,00 & 0,00 & 0,00 & 0,00 \\
\hline Turdus albicollis Vieillot, 1818 & 0,00 & 0,86 & 0,41 & 0,00 & 0,00 & 0,00 & 0,00 & 0,00 \\
\hline Coereba flaveola (Linnaeus, 1758) & 1,22 & 2,86 & 0,00 & 0,44 & 0,00 & 0,00 & 0,54 & 1,16 \\
\hline Saltator similis d'Orbigny \& Lafresnaye, 1837 & 1,83 & 1,43 & 0,41 & 0,44 & 0,67 & 0,00 & 1,63 & 0,39 \\
\hline Pyrrhocoma ruficeps (Strickland, 1844) & 0,00 & 0,00 & 0,00 & 0,44 & 0,00 & 0,00 & 0,00 & 0,00 \\
\hline Tachyphonus coronatus (Vieillot, 1822) & 0,00 & 0,00 & 0,00 & 0,44 & 0,00 & 0,00 & 0,00 & 0,78 \\
\hline Lanio melanops (Vieillot, 1818) & 0,00 & 0,00 & 0,00 & 0,00 & 0,00 & 0,00 & 0,00 & 0,78 \\
\hline Tangara sayaca (Linnaeus, 1766) & 0,20 & 1,43 & 0,00 & 0,00 & 0,00 & 0,00 & 0,00 & 0,00 \\
\hline Tangara preciosa (Cabanis, 1850) & 0,00 & 1,14 & 0,00 & 0,00 & 0,00 & 0,00 & 0,00 & 0,00 \\
\hline Stephanophorus diadematus (Temminck, 1823) & 0,81 & 0,00 & 0,00 & 0,00 & 0,00 & 0,00 & 0,00 & 0,00 \\
\hline Pipraeidea bonariensis (Gmelin, 1789) & 0,00 & 0,00 & 0,00 & 0,89 & 0,00 & 0,00 & 0,00 & 0,00 \\
\hline Conirostrum speciosum (Temminck, 1824) & 0,00 & 0,57 & 0,00 & 1,78 & 0,00 & 0,00 & 0,00 & 0,00 \\
\hline Zonotrichia capensis (Statius Muller, 1776) & 1,63 & 2,29 & 0,83 & 0,00 & 0,67 & 1,44 & 1,09 & 1,16 \\
\hline Poospiza lateralis (Nordmann, 1835) & 0,00 & 0,29 & 0,00 & 0,00 & 0,00 & 0,96 & 0,00 & 0,00 \\
\hline $\begin{array}{l}\text { Cyanoloxia glaucocaerulea (d'Orbigny \& } \\
\text { Lafresnaye, 1837) }\end{array}$ & 0,41 & 0,00 & 0,00 & 0,00 & 0,00 & 0,00 & 0,00 & 0,00 \\
\hline Cyanoloxia brissonii (Lichtenstein, 1823) & 0,00 & 0,00 & 0,00 & 0,00 & 0,00 & 0,96 & 0,00 & 0,00 \\
\hline Parula pitiayumi (Vieillot, 1817) & 0,61 & 0,86 & 1,24 & 0,00 & 0,00 & 0,48 & 0,00 & 0,00 \\
\hline Basileuterus culicivorus (Deppe, 1830) & 1,02 & 2,00 & 1,24 & 3,11 & 4,67 & 2,40 & 2,72 & 1,94 \\
\hline Basileuterus leucoblepharus (Vieillot, 1817) & 2,04 & 2,57 & 2,07 & 4,00 & 4,67 & 1,92 & 2,72 & 3,10 \\
\hline Cacicus chrysopterus (Vigors, 1825) & 0,00 & 0,57 & 0,83 & 0,00 & 0,00 & 0,00 & 0,00 & 0,00 \\
\hline Icterus cayanensis (Linnaeus, 1766) & 0,00 & 0,29 & 0,00 & 0,00 & 0,00 & 0,00 & 0,00 & 0,00 \\
\hline
\end{tabular}

Threatened - espécie que não está ameaçada no presente, mas corre risco de ficar ameaçada num futuro próximo). Conforme se nota, três das quatro espécies constantes no Livro Vermelho foram encontradas no Fragmento 8, considerado o melhor em termos de estado de conservação.

O Instituto Ambiental do Paraná (Mikich \& Bérnils, 2004) publicou o Livro Vermelho da Fauna
Ameaçada no Estado do Paraná, em 2004, o qual segue como Lista Oficial do Estado, na tomada de suas decisões quanto à conservação dessas espécies. Ao definir critérios e categorias de ameaça para o Livro Vermelho, aplicaram-se não somente as recomendações indicadas pelo órgão máximo da metodologia de confecção de listas de espécies ameaçadas (IUCN, 2001 apud Mikich \& Bérnils, 2004), mas também a orientação de colaboradores 


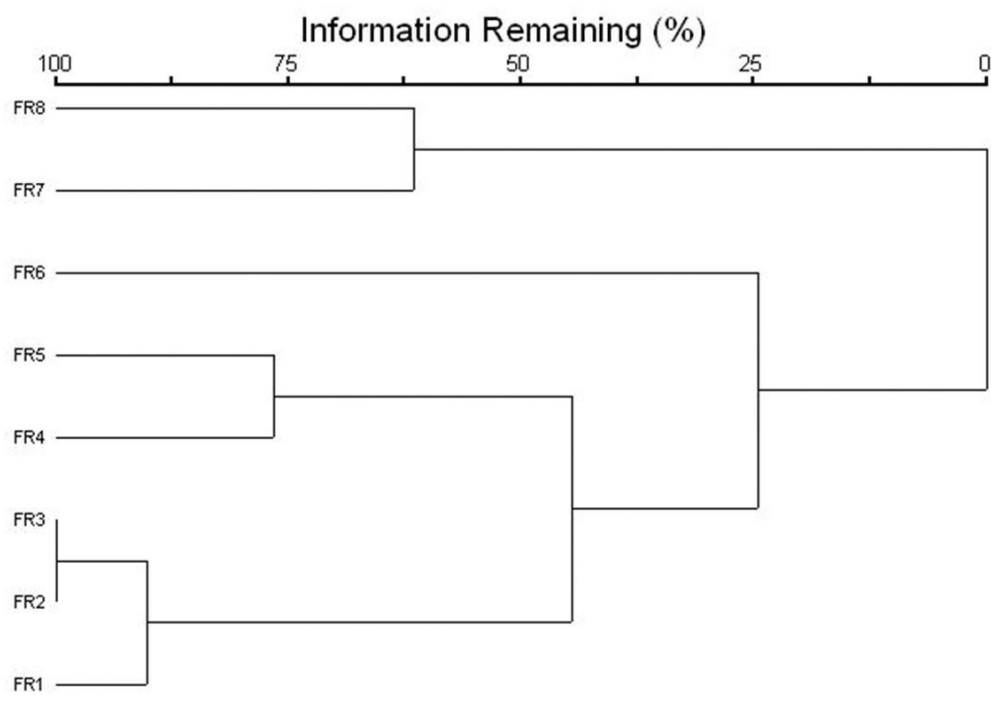

Figura 2. Análise de agrupamento hierárquico de fragmentos com diferentes tamanhos nas fazendas da Emílio B. Gomes em Guamirim, Irati-PR.

Figure 2. Cluster analysis of different size patches in Emílio B. Gomes farms on Guamirim, Irati, PR.

do corpo técnico da Species Survival Commission da IUCN e de seu parceiro institucional, o Conservation International (Gärdenfors et al., 2001 apud Mikich \& Bérnils, 2004). Ainda, na mesma obra:

Objetivando facilitar comparações entre a presente lista paranaense, a atual lista nacional (Brasil, 2003) e outras listas de fauna ameaçada que utilizem as categorias e critérios de IUCN (2001) e Gärdenfors et al. (2001), foi mantida em todos os textos a siglação em língua inglesa adotada nos documentos citados (...). Outra característica relevante da regionalização da análise das espécies ameaçadas é a possibilidade de se efetuar transferências de categorias das listas mundial e nacional para a estadual, na forma de 'upgrades' ou 'downgrades'. Isso fica claro, por exemplo, para espécies que nacionalmente não correm risco e, portanto, não foram citadas na lista brasileira de 2003, mas que em vários estados podem estar seriamente ameaçadas (...). Em posse da versão 3.1 das categorias e critérios da IUCN (2001), cada espécie deve passar por um inquérito que avalia o grau de ameaça a que está sujeita. Esse sistema de inquérito foi estabelecido em IUCN (1994) e aperfeiçoado em IUCN (2001), na tentativa de diminuir a subjetividade das apreciações até então usadas para a definição de espécies ameaçadas. Atualmente são considerados os seguintes critérios básicos: redução do tamanho da população, diminuição da extensão da área de ocorrência ou da área de ocupação (incluindo fragmentação, diminuição continuada e oscilações), número de indivíduos maduros na natureza e análise quantitativa mostrando a probabilidade de extinção na natureza em relação ao tempo ou ao número de gerações (Mikich \& Bérnils, 2004).
Nenhum dos fragmentos amostrados pode ser considerado mata primária, pois todos sofreram algum tipo de intervenção antrópica, em maior ou menor grau, sendo atualmente classificados como de vegetação secundária por simplificação (quando não houve corte raso, mas a retirada seletiva de madeira, caça e retirada de outros produtos vegetais).

Os fragmentos em pior estado de conservação parecem ser os da Fazenda Arroio Grande III, já que estes apresentaram menor tamanho (22,03 ha no total) e uma das menores riquezas (31 espécies).

Alguns dos resultados e vantagens da parceria entre a empresa e a academia, visando à certificação florestal por um lado e a pesquisa científica por outro, foram publicados por Marcelino \& Miskalo (2012).

\section{CONCLUSÕES}

Ficou claro que os melhores fragmentos em termos de estado de conservação - e, consequentemente, com maior valor de conservação - são aqueles pertencentes à Fazenda Água Quente I, em especial o Fragmento 8. A indicação de valor de conservação aqui é dada pelas espécies mais exigentes em termos de habitat florestal.

A presença das espécies florestais, indicadoras de bom estado de conservação (e respectivas quantidades 
de indivíduos registrados) nos fragmentos 7 e 8 , se deve: a) ao tamanho dos fragmentos, bem maiores do que os demais; portanto, com núcleo florestal maior; b) ao melhor estado de conservação da vegetação original, a Floresta Ombrófila Mista Montana, o que pode ser constatado no relatório do inventário arbóreo.

Sugere-se, como primeira prática de manejo, enriquecer os demais fragmentos com o plantio de espécies típicas da FOM, especialmente as frutíferas, de modo a atrair e manter a fauna silvestre. A vigilância dessas áreas e a educação ambiental para os moradores vizinhos também são ações a serem consideradas para garantir a perpetuação desses fragmentos e a regeneração de sua vegetação.

Sugere-se também que todos os funcionários sejam conscientizados sobre a importância da manutenção da araucária em áreas naturais protegidas, a saber: a) para a manutenção da própria espécie e seu ecossistema, a Floresta de Araucária; b) para a manutenção da fauna, que depende dos pinhões para sua alimentação. Também devem ser conscientizados sobre a proibição da coleta de pinhões em áreas naturais antes da data estipulada pelo órgão ambiental competente, que leva em consideração a alimentação da fauna silvestre em época de escassez de alimento na natureza.

Ainda quanto à conscientização dos funcionários - e também visitantes -, é importante o conhecimento sobre a proibição de caça e perseguição de espécies da fauna silvestre, e a sua importância, além da manutenção/recuperação de corredores ecológicos para ligar fragmentos florestais.

A retirada de erva-mate das Reservas Legais deve ser controlada, de modo a não danificar em demasia e desnecessariamente as demais formas de vegetação, e a não atingir as Áreas de Preservação Permanente.

É interessante a criação de um projeto de educação e conscientização ambiental para a comunidade adjacente às áreas da fazenda. O mesmo pode ser feito com os familiares dos funcionários diretos e indiretos, em dias específicos para isso, talvez em forma de visita coletiva com palestras e caminhadas educativas.

As espécies registradas nos fragmentos florestais são apenas uma amostra da comunidade de aves que possa existir na mata nativa, além daquelas que frequentam preferencialmente as áreas antropizadas, necessitando de mais estudos para um levantamento exaustivo nas fazendas.

\section{AGRADECIMENTOS}

A empresa Emílio B. Gomes, pelo apoio financeiro à pesquisa; ao Diretor Carlos Branco e ao Engenheiro Florestal Agnaldo Mattos, pelo contato inicial e constante apoio logístico e administrativo; aos Técnicos Florestais Márcio Gureski e Samuel Garzuze de Mello, e ao Sr. João Maria Pereira, que constantemente nos acompanharam nas muitas trilhas e estradas percorridas pelas fazendas, além de outros que, de alguma forma, nos ajudaram no trabalho. À UNICENTRO, pela parceria estabelecida. Ao Prof. Dr. Antonio José de Araujo, pela nossa indicação e intermediação inicial com a empresa.

\section{STATUS DA SUBMISSÃO}

Recebido: 19 jul., 2012

Aceito: 26 fev., 2014

Publicado: 30 jun., 2014

\section{AUTOR(ES) PARA CORRESPONDÊNCIA}

\section{Vânia Rossetto Marcelino}

Departamento de Engenharia Florestal - DEF, Universidade Estadual do Centro-

Oeste - UNICENTRO, BR 153, Km 07, Bairro Riozinho, CEP 84500-000, Irati, PR, Brasil e-mail: vania@irati.unicentro.br

\section{Kelly Geronazzo Martins}

Departamento de Engenharia

Ambiental - DENAM, Universidade Estadual do Centro-Oeste - UNICENTRO, BR 153, Km 07, Bairro Riozinho, CEP 84500-000, Irati, PR, Brasil e-mail: kellygm77@gmail.com

\section{APOIO FINANCEIRO}

Emílio B. Gomes \& Filhos S/A Indústria, Comércio e Exportação de Madeiras. 


\section{REFERENNCIAS}

Brasil. Instrução Normativa $n^{\circ}$ 03/2003. Lista das espécies ameaçadas no Brasil. Brasília: IBAMA; 2003.

Castella PR, Britez RM. A floresta com araucária no Paraná: conservação e diagnóstico dos remanescentes florestais. Brasília: Ministério do Meio Ambiente; 2004.

Comitê Brasileiro de Registros Ornitológicos - CBRO. Listas das aves do Brasil. 2011. [cited 2012 maio 20]. Available from: http://www.cbro.org.br.

Fundação de Pesquisas Florestais do Paraná - FUPEF. Aspectos faunísticos da Floresta Nacional de Irati. Projeto Manejo de Florestas Nacionais. Curitiba: IBAMA/ FUPEF; 1990.

Gärdenfors UC, Hilton-Taylor GM, Rodriguez JP. The application of IUCN red list criteria at regional levels. Conservation Biology 2001; 15(5): 1206-1212. http:// dx.doi.org/10.1046/j.1523-1739.2001.00112.x

International Union for Conservation of Nature and Natural Resources - IUCN. IUCN Red List Categories and Criteria Version 3.1. Gland \& Cambridge: IUCN Species Survival Commission. 2001.

International Union for Conservation of Nature and Natural Resources - IUCN. IUCN Red List Categories. Gland \& Cambridge: IUCN Species Survival Commission; 1994.

Laurance WF. Have we overstated the tropical biodiversity crisis? Trends in Ecology and Evolution 2007; 22(2): s65-70. PMid:17011069. http://dx.doi. org/10.1016/j.tree.2006.09.014

Marcelino VR, Adenesky-Filho E. Aves e mamíferos registrados no Colégio Florestal de Irati, Paraná. In: Anais da X Semana de Estudos Florestais e I Seminário de Atualização Florestal: Florestas de Produção; 2008; Irati. Guarapuava: Universidade Estadual do CentroOeste; 2008. CD-ROM.

Marcelino VR, Miskalo, EM. Um projeto de extensão como forma de parceria entre empresa e universidade. In: Anais do 50 Salão de Extensão e Cultura da UNICENTRO; 2012; Irati. Guarapuava: Universidade Estadual do Centro-Oeste; 2012. v. 3, n. 1. Available from: http://anais.unicentro.br/sec/vsec/.

Marcelino VR, Romaniuk DS. Levantamento complementar das aves da Floresta Nacional de Irati, PR. In: Anais do II Seminário de Atualização Florestal e XI Semana de Estudos Florestais; 2010; Irati. Guarapuava: Universidade Estadual do Centro-Oeste; 2010. CD-ROM.
Marcelino VR, Silva MM. Aves e mamíferos registrados no Campus de Irati da Unicentro. In: Anais da X Semana de Estudos Florestais e I Seminário de Atualização Florestal: Florestas de Produção; 2008; Irati. Guarapuava: Universidade Estadual do Centro-Oeste; 2008. CDROM.

McCune B, Grace JB. Analysis of Ecological Communities. Oregon: MJM; 2002.

Mikich SB, Bérnils RS. Livro Vermelho da Fauna Ameaçada no Estado do Paraná. 2004. [cited 2004 ago. 02]. Available from: http://www.pr.gov.br/iap.

Ribeiro MC, Metzger JP, Martensen AC, Ponzoni FJ, Hirota MM. The Brazilian Atlantic Forest: how much is left, and how is the remaining forest distributed? Implications for conservation. Biological Conservation 2009; 142: 1141-1153. http://dx.doi.org/10.1016/j. biocon.2009.02.021

Romaniuk DS. Comparação da avifauna amostrada com rede-neblina em duas áreas florestais na FLONA de Irati, Paraná [monografia]. Irati: Universidade do Centro Oeste do Paraná; 2011.

Romaniuk DS, Winagraski E, Marcelino VR. Comparação qualitativa da avifauna de duas áreas com parcelas permanentes na Floresta Nacional de Irati usando redes-neblina como método amostral. In: Anais da II Semana de Integração Ensino, Pesquisa e Extensão; 2011; Irati. Guarapuava: Editora da UNICENTRO; 2011. Available from: http://anais.unicentro.br/siepe/2011/.

Volpato GH. Comunidades de aves em mosaico de habitat formado por Floresta Ombrófila Mista e plantações com Araucaria angustifolia e com Pinus elliottii, no sul do estado do Paraná, Brasil [tese]. Curitiba: Universidade Federal do Paraná; 2009.

Volpato GH, Prado VM, Anjos L. What can tree plantations do for forest birds in fragmented forest landscapes? A case study in southern Brazil. Forest Ecology and Management 2010; 260: 1156-1163. http:// dx.doi.org/10.1016/j.foreco.2010.07.006

Winagraski E, Romaniuk DS, Marcelino VR. Amostragem da avifauna na Estação Ecológica de Fernandes Pinheiro utilizando redes-neblina. In: Anais da II Semana de Integração Ensino, Pesquisa e Extensão. Irati. Guarapuava: Editora da UNICENTRO; 2011. Available from: http://anais.unicentro.br/siepe/2011/.

Zar JH. Biostatistical analysis. New Jarsey: PrenticeHall; 1999. 